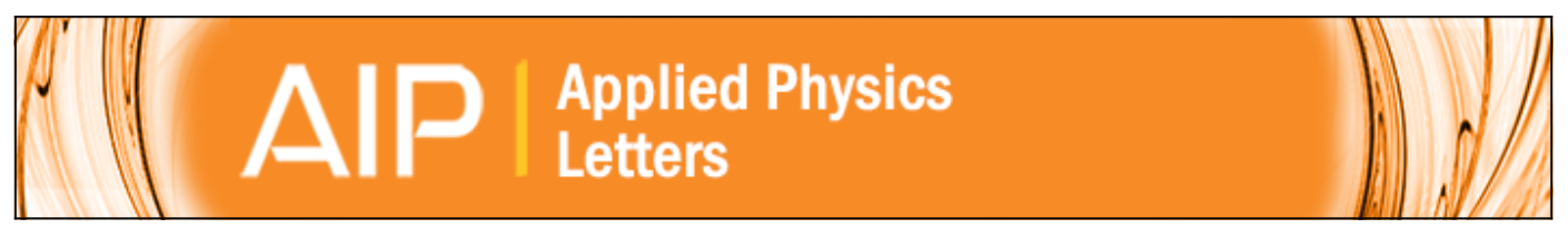

\title{
Charge transport model in nanodielectric composites based on quantum tunneling mechanism and dual-level traps
}

Guochang Li, George Chen, and Shengtao Li

Citation: Applied Physics Letters 109, 062901 (2016); doi: 10.1063/1.4960638

View online: http://dx.doi.org/10.1063/1.4960638

View Table of Contents: http://scitation.aip.org/content/aip/journal/apl/109/6?ver=pdfcov

Published by the AIP Publishing

\section{Articles you may be interested in}

Charge transport in molecular junctions: From tunneling to hopping with the probe technique

J. Chem. Phys. 143, 024111 (2015); 10.1063/1.4926395

Room temperature positive magnetoresistance via charge trapping in polyaniline-iron oxide nanoparticle composites

Appl. Phys. Lett. 103, 032408 (2013); 10.1063/1.4815998

Collective charge transport in semiconductor-metal hybrid nanocomposite

Appl. Phys. Lett. 102, 053107 (2013); 10.1063/1.4790300

Tunneling conductivity in composites of attractive colloids

J. Chem. Phys. 136, 164903 (2012); 10.1063/1.4705307

Charge transport in polypyrrole:ZnO-nanowires composite films

Appl. Phys. Lett. 95, 202106 (2009); 10.1063/1.3266525

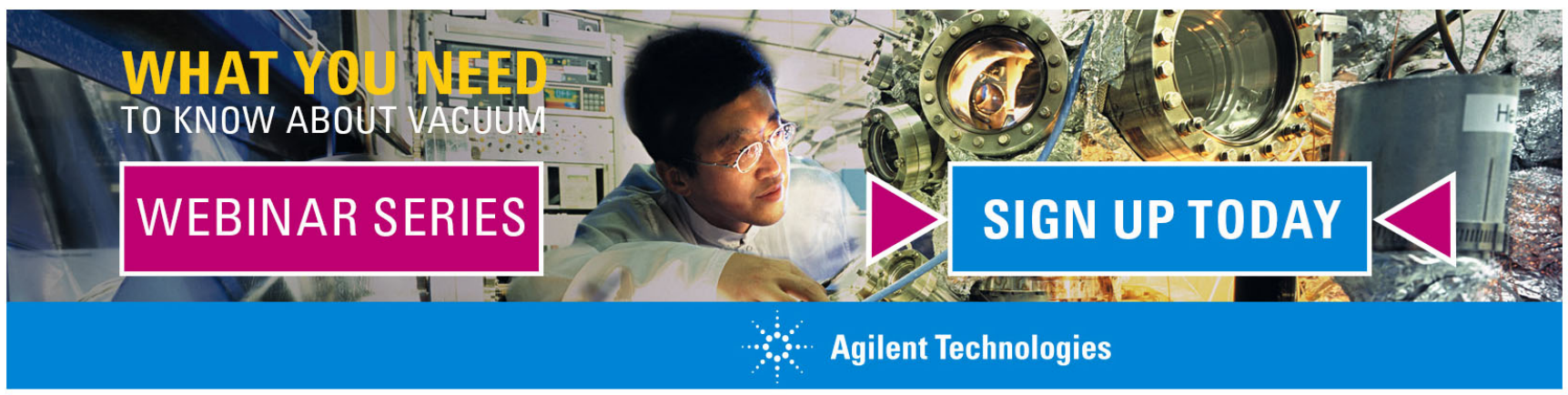




\title{
Charge transport model in nanodielectric composites based on quantum tunneling mechanism and dual-level traps
}

\author{
Guochang Li, ${ }^{1,2}$ George Chen, ${ }^{1,2, a)}$ and Shengtao Li ${ }^{1, a)}$ \\ ${ }^{1}$ State Key Laboratory of Electrical Insulation and Power Equipment, Xi' an Jiaotong University, \\ $X i$ ' an 710049, China \\ ${ }^{2}$ School of Electronic and Computer Science, University of Southampton, Southampton SO17 1 BJ, \\ United Kingdom
}

(Received 22 June 2016; accepted 28 July 2016; published online 8 August 2016)

\begin{abstract}
Charge transport properties in nanodielectrics present different tendencies for different loading concentrations. The exact mechanisms that are responsible for charge transport in nanodielectrics are not detailed, especially for high loading concentration. A charge transport model in nanodielectrics has been proposed based on quantum tunneling mechanism and dual-level traps. In the model, the thermally assisted hopping (TAH) process for the shallow traps and the tunnelling process for the deep traps are considered. For different loading concentrations, the dominant charge transport mechanisms are different. The quantum tunneling mechanism plays a major role in determining the charge conduction in nanodielectrics with high loading concentrations. While for low loading concentrations, the thermal hopping mechanism will dominate the charge conduction process. The model can explain the observed conductivity property in nanodielectrics with different loading concentrations. Published by AIP Publishing. [http://dx.doi.org/10.1063/1.4960638]
\end{abstract}

Nanodielectrics have been widely investigated in the last two decades as some improved properties, including space charge suppression, corona resistance, and partial discharge resistance, have been observed. Especially, space charge suppression when the nanodielectrics are subjected to the applied dc field has an important significance for applications in high voltage dc insulation system.

It has been widely reported that the formation of deep traps between the polymer matrix and nanoparticles can suppress space charge injection. ${ }^{1,2}$ However, the effect of space charge suppression has a strong link with the nanoparticle concentration. For low loading concentration, the charge suppression can be observed clearly by the pulsed electroacoustic method (PEA), while charge transport behavior becomes more complicated for high loading concentration. ${ }^{3}$ Literature reports and our own measurement results indicated that the conductivity properties in nanodielectrics show different changes for different loading concentrations, ${ }^{3-8}$ as shown in Figure 1. The polymer matrixs are LDPE (Low density polyethylene), LLDPE (Linear low density polyethylene), and PI (Polyimide), respectively. One may notice that the conductivity for polyethylene without any nanofillers shows the difference in several orders of magnitude. This is likely related to the purity of the sample, sample preparation, the influence of test method and test environment, etc. But for each group where the same test setup, method, and samples are used, the conductivity results show the expected tendency. More importantly, the following trend has been observed for all the samples. For the nanodielectrics with low concentration, the conductivity is lower than that of the pure polymer. It reaches a minimum value at a certain concentration and gradually becomes large with the increase of loading concentration. It has also been found that the related electrical performance, surface flashover, and breakdown deteriorate for

a)Electronic addresses: gc@ecs.soton.ac.uk and sli@mail.xjtu.edu.cn nanodielectrics with high concentrations. ${ }^{3,4,8,9}$ At present, the exact mechanisms of charge transport in nanodielectrics are not detailed, and the observed experiment phenomenon of charge conduction in nanodielectrics with high loading concentrations has never been fully explained.

Adding nanoparticles into polymers can introduce the deep traps due to the effect of interfacial characteristics between the nanoparticle and matrix, and the deep trap density is different for different loading concentrations. ${ }^{1,2}$ Generally, it is difficult for charge carriers to escape from the deep trap sites, due to the higher potential barrier, especially for nanodielectrics with high loading concentration, which is inconsistent with the observed conductivity phenomenon. The quantum tunneling mechanism based on the percolation theory has been investigated in black carbon and carbon nanotube polymer

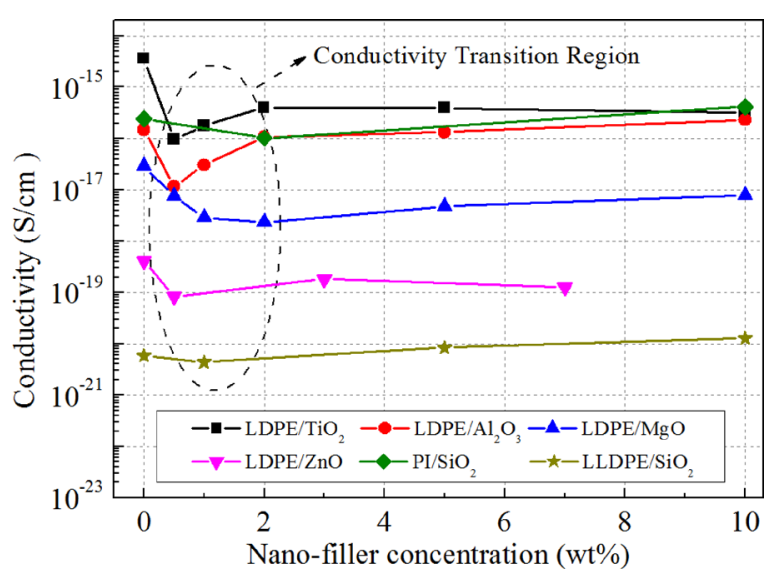

FIG. 1. Conductivity characteristics of nanodielectrics as a function of loading concentrations. (a) $\mathrm{LDPE} / \mathrm{TiO}_{2}-50 \mathrm{kV} / \mathrm{mm}-25^{\circ} \mathrm{C}$, black square original data from Ref. 6; (b) $\mathrm{LDPE} / \mathrm{Al}_{2} \mathrm{O}_{3}-40 \mathrm{kV} / \mathrm{mm}-25^{\circ} \mathrm{C}$, red circle original data from Ref. 8; (c) LDPE/MgO- $40 \mathrm{kV} / \mathrm{mm}-25^{\circ} \mathrm{C}$, blue triangle original data from Ref. 4; (d) $\mathrm{LDPE} / \mathrm{ZnO}-40 \mathrm{kV} / \mathrm{mm}-25^{\circ} \mathrm{C}$, inverted pink triangle original data from Ref. 5; (e) $\mathrm{PI} / \mathrm{SiO}_{2}-40 \mathrm{kV} / \mathrm{mm}-100^{\circ} \mathrm{C}$, green diamond original data from Ref. 7; (f) $\mathrm{LLDPE} / \mathrm{SiO}_{2}-50 \mathrm{kV} / \mathrm{mm}-60^{\circ} \mathrm{C}$, green star original data from Ref. 3. 
composites. ${ }^{10,11}$ We believe that it can also be used to represent the charge conduction in nanodielectrics loaded with metallic oxide. For high loading concentration, the separation distance between the two adjacent nanoparticles is short. Hence, it is possible that the trapped charges pass through the trap site to the nearest-neighbor site by the quantum tunneling effect.

In this paper, a charge transport model in nanodielectrics has been proposed based on quantum tunneling mechanism and dual-level traps to interpret the observed charge conduction in nanodielectrics with different loading concentrations. Figure 2 shows the schematic model of charge transport in nanodielectrics.

In the model, dual-level traps, that is, shallow trap and deep trap are taken into account. ${ }^{12}$ There are two kinds of charge transport mechanisms, which can represent the charge conduction processes for the shallow trap and the deep trap, respectively. For the shallow traps, the trapped charges can overcome the potential barrier and hop to the neighbour trip site by the effect of thermal vibration, which depends on the temperature and electric field. Hence, thermally assisted hopping process is considered to represent the charge conduction in the shallow traps. For the deep traps, it is difficult for the trapped charges to overcome the potential barrier. But there is the exception that if the electric field is strong enough, and the distance of potential barrier between two trap sites is small enough, trapped charges are able to pass through the potential well by the quantum tunneling mechanism. In the calculation, assumptions include injection barriers for electrons and holes to be symmetrical, and nanoparticles with the same diameter are uniformly dispersed in the polymer matrix.

The injected charges from electrodes will migrate to the bulk of nanodielectrics under the effect of the electric field. Neglecting the diffusion process, charge transport dynamic behaviors in nanodielectrics can be represented by the following expressions:

Charge transport equation

$$
j_{e, h}(x, t)=\rho_{e, h}(x, t) \mu_{e, h}(x, t) F(x, t) .
$$

Poisson's equation

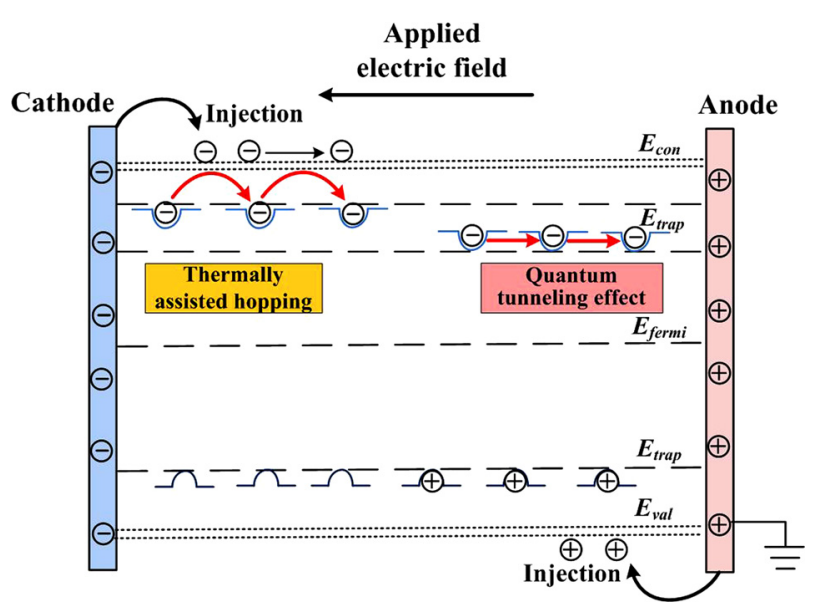

FIG. 2. Schematic model of charge transport in nanodielectrics.

$$
\frac{\partial F(x, t)}{\partial x}=\frac{\rho_{\text {total }}(x, t)}{\varepsilon_{0} \varepsilon_{\mathrm{r}}} .
$$

Charge continuity equation

$$
\frac{\partial \rho_{\text {total }}(x, t)}{\partial t}+\frac{\partial j_{e, h}(x, t)}{\partial x}=S_{i}
$$

where $j_{e, h}(x, t)$ is the conduction current density of electrons and holes, $\mathrm{A} / \mathrm{m}^{2} ; \rho_{e, h}(x, t)$ is the free charge density and $\rho_{\text {total }}(x, t)$ is the total charge density, $\mathrm{C} / \mathrm{m}^{3} ; \mu_{e, h}(x, t)$ is the mobility of electrons and holes, $\mathrm{m}^{2} /(\mathrm{V} \mathrm{s}) ; F(x, t)$ is the electric field, $\mathrm{V} / \mathrm{m} ; S_{i}$ is the source term, $\mathrm{m}^{3} \mathrm{C}^{-1} \mathrm{~s}^{-1}$; here, it refers to the recombination of the total electrons and total holes.

The Schottky law is adopted to describe the charge injection process from electrode to dielectric. The injection current density depends on the contact potential barrier, the applied voltage, and temperature

$J_{\text {in_e }}(0, t)=A T^{2} \exp \left[-\frac{\left(q_{e} E_{\text {in }(e)}-\sqrt{q_{e}^{3} F(0, t) / 4 \pi \varepsilon_{0} \varepsilon_{r}}\right)}{k_{B} T}\right]$,

$J_{\text {in } \_h}(d, t)=A T^{2} \exp \left[-\frac{\left(q_{e} E_{\text {in }(h)}-\sqrt{q_{e}^{3} F(d, t) / 4 \pi \varepsilon_{0} \varepsilon_{r}}\right)}{k_{B} T}\right]$,

where $j_{i n \_e}$ and $j_{i n \_} h$ are injection current density from cathode and anode, respectively, $\mathrm{A} / \mathrm{m}^{2} ; E_{i n(e)}$ and $E_{i n(h)}$ are the injection barriers for electrons and holes, respectively, $\mathrm{eV}$; $A$ is the Richardson constant; $T$ is the absolute temperature, $\mathrm{K} ; q_{e}$ is the elementary electron, $\mathrm{C} ; h$ is the Planck constant, and $k_{B}$ is the Boltzmann constant; $\varepsilon_{0}$ is the vacuum permittivity, $\mathrm{F} / \mathrm{m}$, and $\varepsilon_{r}$ is the relative permittivity of nanodielectrics.

Thermally assisted hopping conductivity (TAH) originally developed for charge transportation in ionic crystals, which can represent the process of thermal excitation of trapped charges in the shallow traps. The expressions are as follows: ${ }^{13}$

$$
\sigma_{\mathrm{TAH}}(F, T)=\left[\left(\frac{2 N v_{\mathrm{TAH}} q_{e} a}{F}\right) \exp \left(\frac{-\Delta H}{k_{\mathrm{B}} T}\right) \sinh \left(\frac{a q_{e} F}{2 k_{\mathrm{B}} T}\right)\right],
$$

where $N$ is the carrier density, $\mathrm{m}^{-3} ; a$ is the average separation distance, $\mathrm{nm} ; v_{T A H}$ is the frequency of hopping; $\triangle H$ is the trap energy, eV.

The quantum tunneling conductivity (TUN) was initially developed by Mott and Davis, and extended by Apsley and Hughes. ${ }^{14}$ Charge transport processes are composed of a series of tunneling processes; hence, the overall conductivity depends on an average of the probabilities of sequential tunneling. The geometric mean is used to describe the process ${ }^{14}$

$$
\langle P\rangle=\lim _{n \rightarrow \infty}\left[\prod_{i}^{n} P_{i}\right]^{1 / n}=\exp \left[\lim _{n \rightarrow \infty} \frac{1}{n} \sum_{i}^{n} \ln P_{i}\right]
$$


where $P_{i}$ is the probability of an individual tunneling process. After complex mathematics, the tunneling conductivity can be represented as follows:

$$
\begin{aligned}
\sigma_{T U N}(F, T) \cong & \frac{N k_{B} T v_{p h} q_{e}}{2 \alpha F}\left[1+\frac{P+Q}{P+1}\right]\left[\frac{2}{K(P+Q)}\right]^{1 / 4} \\
& \times\left[\frac{\frac{3+\beta}{24(1+\beta)^{3}}-\frac{1}{8}-\frac{\beta}{3}}{\frac{2+\beta}{6(1+\beta)^{2}}+\frac{1}{3}+\frac{\beta}{2}}\right] \\
& \times \exp \left[-\left(\frac{2}{K(P+Q)}\right)^{1 / 4}\right],
\end{aligned}
$$

where $\beta$ is the ratio of field energy to thermal energy

$$
\beta=\frac{q_{e} F}{2 \alpha k_{B} T},
$$

and

$$
K=\frac{N \pi k_{B} T}{24 \alpha^{3}}, \quad P=\frac{1+\beta / 2}{(1+\beta)^{2}}, \quad Q=\frac{3 \beta}{2}+1,
$$

where $K, P$, and $Q$ are the intermediate variables to simplify the equation; $N$ is the energy density of trap states, $\mathrm{m}^{-3} ; \alpha$ is the decay constant of localized state wave functions. The average trap separation distance of $d$ equals to $(2 \alpha)^{-1}, \mathrm{~nm}$.

Interfacial zone between the polymer matrix and nanoparticles plays a key role in determining charge transport behaviors and will have a significant effect on the electrical performance of nanodielectrics. Several models have been proposed to characterize the properties of interfacial zone, ${ }^{9,15,16}$ such as, multi-region structure around spherical nanoparticles model. These proposed models are beneficial to understand the interfacial characteristics and some observed experimental phenomena. However, it is difficult to gauge or calculate the actual thickness of the interfacial zone, due to the complexity of interfacial zone. Hence, the separation distance between the two adjacent nanoparticles cannot be determined definitely as there is no specific value of thickness of interfacial zone for different loading concentrations.

In the simulation, nanoparticle loading concentration is not adopted directly. Instead, the trap energy and separation distance between the two adjacent trap sites are discussed, which has a qualitative link to the loading concentration. Dual-level traps are considered in the calculation. The values of shallow trap and deep trap are set to $0.76 \mathrm{eV}$ and $1.10 \mathrm{eV}$, respectively, based on previous studies of LDPE or nanodielectrics $\left(0.8-1.0 \mathrm{eV},{ }^{1,2} 0.76 \mathrm{eV},{ }^{13} 0.88 \mathrm{eV}\right.$, and $1.01 \mathrm{eV} .^{12}$ The separation distance between the two adjacent trap sites is set from 0.1 to $5 \mathrm{~nm}$, which is extracted from the experimental results of LDPE or nanodielectrics $(1.1 \mathrm{~nm}$ and $2.9 \mathrm{~nm},{ }^{13} 3.2 \mathrm{~nm},{ }^{17} 2.8 \mathrm{~nm}^{18}$ ). In the simulation, a voltage of $9 \mathrm{kV}$ is applied on the nanodielectrics with a thickness of $180 \mu \mathrm{m}$. The whole sample was discretized into 200 elements. Considering the volt-on time in PEA measurement, the total calculation time is set to $1 \mathrm{~h}$ and the time interval is $0.1 \mathrm{~s}$. The numerical calculation has carried out by Finite Difference Method (FDM). A summary of the parameters of simulation is presented in Table I.
TABLE I. Definition of parameters in simulation.

\begin{tabular}{lc}
\hline \hline Model parameter & Value \\
\hline $\mathrm{T}$, Temperature (K) & 300 \\
$F$, Applied electric field $(\mathrm{kV} / \mathrm{mm})$ & 50 \\
$E_{\text {in }(e)}$, Injection barrier $(\mathrm{eV})$, for electrons & 1.10 \\
$E_{\text {in }(h)}$, Injection barrier $(\mathrm{eV})$, for holes & 1.10 \\
$E_{\text {shallow }}$, Shallow trap energy (eV), & 0.76 \\
for electrons and holes & \\
$E_{\text {deep }}$, Deep trap energy $(\mathrm{eV})$, & 1.10 \\
for electrons and holes & \\
$a$, Separation distance $(\mathrm{nm})$, for TAH & $0.1-5$ \\
$d$, Separation distance $(\mathrm{nm})$, for TUN & $0.1-5$ \\
$S_{i}$, Recombination coefficient $\left(\mathrm{m}^{3} \mathrm{C}^{-1} \mathrm{~s}^{-1}\right)$, & $4 \times 10^{-3}$ \\
for total electrons and total holes & \\
\hline \hline
\end{tabular}

Space charge and electric field distributions versus sample position and time in nanodielectrics with a certain separation distance are calculated, as shown in Figures 3 and 4. The separation distance is set to $3.2 \mathrm{~nm}$, which was extracted from experimental results of surface potential decay. ${ }^{17}$

It can be observed from Figure 3 that electrons and holes injected from the two electrodes gradually migrate to the bulk of the sample with time, and they will go through a recombination process inside the sample. At the initial stage of stress, most injected charges will be captured by the trap

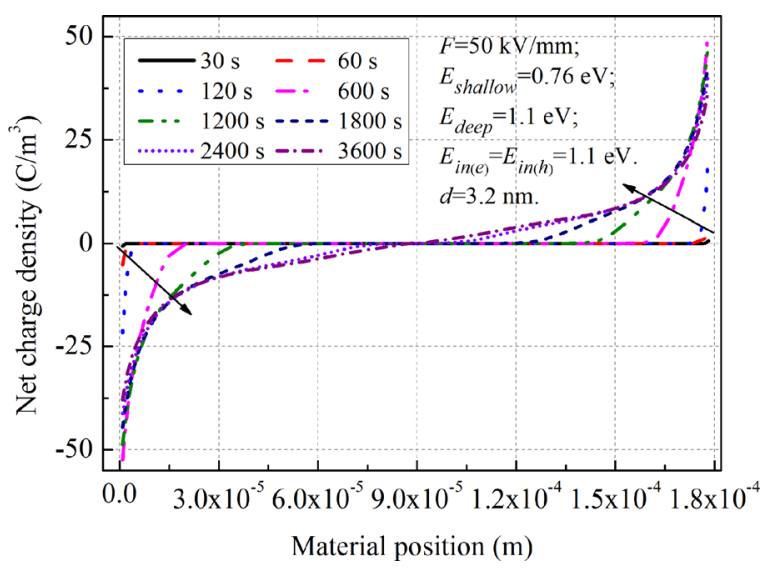

FIG. 3. Net space charge distribution versus sample position and time in nanodielectrics.

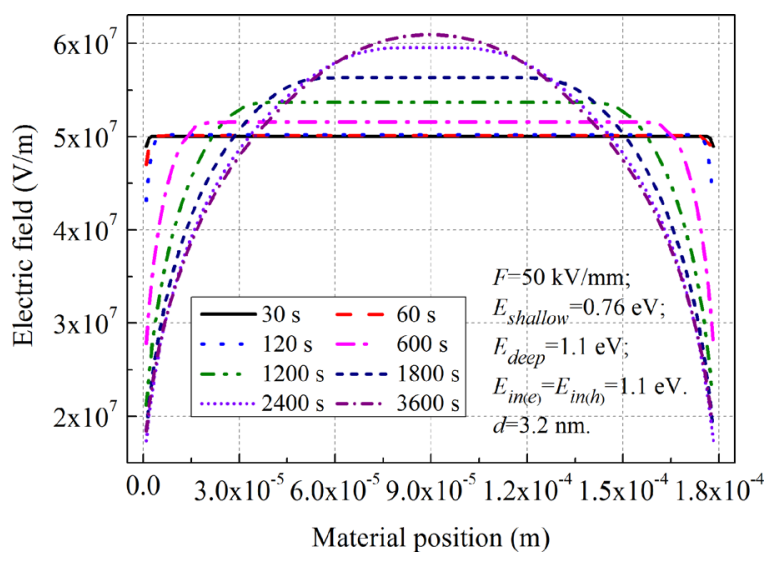

FIG. 4. Electric field distribution versus sample position and time in nanodielectrics. 


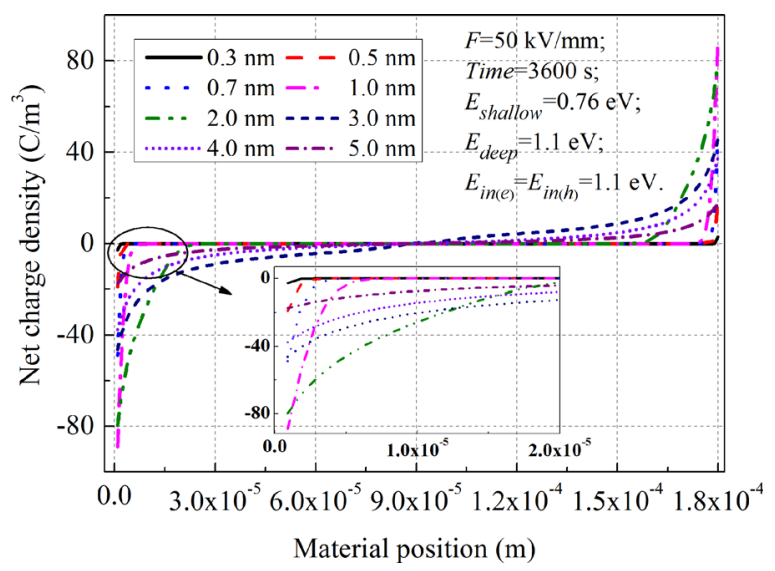

FIG. 5. Net charge distribution versus sample position in nanodielectrics with different separation distances.

sites nearby the electrode interface. Charges at the electrode interface gradually increase with time, and reach a peak, about $52.5 \mathrm{C} / \mathrm{m}^{3}$ at $600 \mathrm{~s}$, which will form an opposite space charge electric field, resulting in the reduction of total electric field. The rate of charge injection will become slow with the decreasing local electric field, but the charge injection process still takes place as long as the electric field exceeds the injection threshold. Overall, the accumulated charges in the bulk of sample gradually increase until they reach a stable state. Consequently, the maximum electric field increases from $5 \times 10^{7} \mathrm{~V} / \mathrm{m}$ to $6.09 \times 10^{7} \mathrm{~V} / \mathrm{m}$ in the bulk of sample under the stress time from $30 \mathrm{~s}$ to $3600 \mathrm{~s}$, as shown in Figure 4.

To consider the influence of the nanoparticle loading concentration on charge transport in nanodielectrics, the influences of the separation distance between the two adjacent trap sites on charge dynamics and conductivity have been studied. Figure 5 shows net charge distributions in nanodielectrics at 3600 s. Figure 6 shows conductivity distribution in nanodielectrics as a function of the separation distance. It can be observed that from Figure 5 the charge distributions present different tendencies with the increase of separation distance, it increases first from $2.9 \mathrm{C} / \mathrm{m}^{3}$ for $0.3 \mathrm{~nm}$ to $89.0 \mathrm{C} / \mathrm{m}^{3}$ for $1.0 \mathrm{~nm}$ and then decreases to $17.5 \mathrm{C} / \mathrm{m}^{3}$ at the cathode interface.

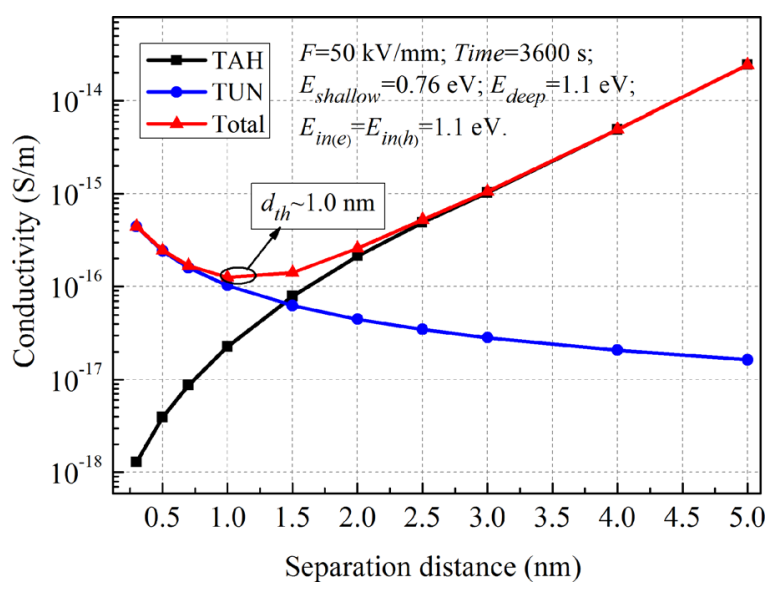

FIG. 6. Conductivity distribution in nanodielectrics as a function of the separation distance.
The conductivity properties are different for nanodielectrics with different separation distances, as shown in Figure 6. It can be seen that the thermal conductivity (TAH) gradually increases from $1.29 \times 10^{-18} \mathrm{~S} / \mathrm{m}$ to $2.44 \times 10^{-14} \mathrm{~S} / \mathrm{m}$ with the increase of separation distance, while tunnelling conductivity (TUN) gradually decreases from $4.44 \times 10^{-16} \mathrm{~S} / \mathrm{m}$ to $1.64 \times 10^{-17} \mathrm{~S} / \mathrm{m}$. The total charge conduction is dependent on the two processes of TAH and TUN, which first decreases and then increases with the increasing of the separation distance. The critical threshold of separation distance is about $1 \mathrm{~nm}$, which is in agreement with the electron tunneling length of polymer composites. ${ }^{11}$

The threshold value may vary within a small range, which depends on the applied electric field, composite structure, and trap distributions. When the separation distance between the two trap sites is shorter than the threshold value, charges may pass through the potential well by the tunneling effect under the strong field; this moment, the thermal hopping process is weak. Overall, the tunneling process dominates the charge conduction, the total conductivity gradually reduces. With the increase of separation distance, the tunneling process gradually becomes difficult, and the thermal hopping process becomes easier. When the separation distance exceeds the threshold value, the thermal hopping process is responsible for the charge conduction and gradually increases with the increase of the separation distance.

The calculation in Figure 6 is consistent with the experimental results in Figure 1. For high loading concentration, the separation distance is short, and it may decrease to several nanometers under the effect of interfacial zone. The tunneling effect may occur between the two nearest nanoparticles, and it will dominate the charge transport process. With the decrease of concentration, the separation distance gradually becomes large, and the tunneling process in nanodielectrics becomes weak. It will result in the decline of the overall conductivity and reach a minimum value. For low loading concentration, the tunneling effect gradually becomes weak, owing to the increase of separation distance. The thermal hopping process becomes easier and will gradually dominate the charge conduction process. So the measured conductivity gradually increases with the decrease of the loading concentrations for the case of low concentration. The explanation is consistent with the bipolar charge transport (BCT) model. The reduction of loading concentration means the decreasing of trap density, so the charge conduction will be accelerated. For the pure polymer, the trap density is less than that of nanodielectric. Hence, the conductivity of pure polymer is higher than that of nanodielectric with low concentration.

In conclusion, in order to explain the observed charge conduction phenomenon and reveal the charge transport mechanism in nanodielectrics, a charge transport model in nanodielectrics has been proposed based on quantum tunneling mechanism and dual-level traps. Distributions of space charge and electric field with sample position and time in nanodielectrics at a certain separation distance, and distributions of space charge and conductivity with different separation distances are calculated. For different loading concentrations, the charge transport mechanisms are different. The quantum tunneling mechanism plays a major role in determining the charge conduction in nanodielectrics with high concentrations. 
While for the case of low concentrations, the thermal hopping mechanism will be dominant, which is identical with the bipolar charge transport (BCT) model. The proposed model is beneficial to explore the electrical performance of nanodielectrics with different nanoparticle loading concentrations.

The authors thank the National Natural Science Foundation of China (Grant Nos. 51337008 and 11575140). G. Li thanks the China Scholarship Council (CSC) for providing the scholarship for visiting Ph.D. studentship at University of Southampton (No. 201506280128).

${ }^{1}$ F. Q. Tian, Q. Q. Lei, X. Wang, and Y. Wang, Appl. Phys. Lett. 99, 142903 (2011).

${ }^{2}$ B. Han, X. Wang, Z. Sun, J. M. Yang, and Q. Q. Lei, Appl. Phys. Lett. 102, 012902 (2013).

${ }^{3}$ G. Chen, S. T. Li, and L. S. Zhong, in International Conference on the Properties and Applications of Dielectric Materials (2015), p. 36.

${ }^{4}$ Y. Murakami, M. Nemoto, S. Okuzumi, S. Masuda, M. Nagao, N. Hozumi, Y. Sekiguchi, and Y. Murata, IEEE Trans. Dielectr. Electr. Insul. 15, 33 (2008).
${ }^{5}$ F. Q. Tian, Q. Q. Lei, X. Wang, and Y. Wang, IEEE Trans. Dielectr. Electr. Insul. 19, 763 (2012).

${ }^{6}$ S. T. Li, N. Zhao, Y. J. Nie, X. Wang, G. Chen, and G. Teyssedre, IEEE Trans. Dielectr. Electr. Insul. 22, 92 (2015).

${ }^{7}$ Y. Cao, P. C. Irwin, and K. Younsi, IEEE Trans. Dielectr. Electr. Insul. 11, 797 (2004).

${ }^{8}$ W. W. Wang, D. M. Min, and S. T. Li, IEEE Trans. Dielectr. Electr. Insul. 23, 564 (2016).

${ }^{9}$ S. T. Li, G. L. Yin, G. Chen, J. Y. Li, S. N. Bai, L. S. Zhong, Y. X. Zhang, and Q. Q. Lei, IEEE Trans. Dielectr. Electr. Insul. 17, 1523 (2010).

${ }^{10}$ W. S. Bao, S. A. Meguid, Z. H. Zhu, and G. J. Weng, J. Appl. Phys. 111, 093726 (2012).

${ }^{11}$ G. Ambrosetti, N. Johner, C. Grimaldi, T. Maeder, P. Ryser, and A. Danani, J. Appl. Phys. 106, 016103 (2009).

${ }^{12}$ T. C. Zhou, G. Chen, R. J. Liao, and Z. Q. Xu, J. Appl. Phys. 110, 043724 (2011).

${ }^{13}$ J. R. Dennison and J. Brunson, IEEE Trans. Plasma Sci. 36, 2246 (2008).

${ }^{14}$ T. Apsley and H. P. Hughes, Philos. Mag. 31, 1327 (1975).

${ }^{15}$ T. J. Lewis, IEEE Trans. Dielectr. Electr. Insul. 11, 739 (2004).

${ }^{16}$ T. Tanaka, M. Kozako, N. Fuse, and Y. Ohki, IEEE Trans. Dielectr. Electr. Insul. 12, 669 (2005).

${ }^{17}$ Z. Ziari, S. Sahli, and A. Bellel, M. J. Condens. Matter 12, 223 (2010).

${ }^{18}$ R. Nath, T. Kaura, and M. M. Perlman, IEEE Trans. Dielectr. Electr. Insul. 25, 419 (1990). 